Research Article

\title{
Revealing the Pharmacological Mechanism of Acorus tatarinowii in the Treatment of Ischemic Stroke Based on Network Pharmacology
}

\author{
FengZhi Liu $\mathbb{D}^{1,2}$ Qian Zhao, ${ }^{3}$ Suxian Liu, ${ }^{4}$ Yingzhi Xu, ${ }^{5}$ Dongrui Zhou, ${ }^{1,2}$ Ying Gao $\mathbb{D}^{5},{ }^{5}$ \\ and Lingqun $\mathrm{Zhu} \mathbb{B}^{1}$ \\ ${ }^{1}$ Key Laboratory of Chinese Internal Medicine of Ministry of Education and Beijing, Dongzhimen Hospital, \\ Beijing University of Chinese Medicine, Beijing 100700, China \\ ${ }^{2}$ Beijing University of Chinese Medicine, Beijing 100029, China \\ ${ }^{3}$ Pharmaceutical Science, Beijing University Health Science Center, Beijing 100089, China \\ ${ }^{4}$ Longhua Hospital Shanghai University of Traditional Chinese Medicine, Shanghai 200032, China \\ ${ }^{5}$ Key Office of Encephalopathy TCM Research, Dongzhimen Hospital, Beijing University of Chinese Medicine, \\ Beijing 100700, China
}

Correspondence should be addressed to Ying Gao; gaoying973@126.com and Lingqun Zhu; wns898@sina.com

Received 2 July 2020; Revised 15 September 2020; Accepted 26 September 2020; Published 31 October 2020

Academic Editor: Rômulo Dias Novaes

Copyright ( $\odot 2020$ FengZhi Liu et al. This is an open access article distributed under the Creative Commons Attribution License, which permits unrestricted use, distribution, and reproduction in any medium, provided the original work is properly cited.

Aim. Stroke is the second significant cause for death, with ischemic stroke (IS) being the main type threatening human being's health. Acorus tatarinowii (AT) is widely used in the treatment of Alzheimer disease, epilepsy, depression, and stroke, which leads to disorders of consciousness disease. However, the systemic mechanism of AT treating IS is unexplicit. This article is supposed to explain why AT has an effect on the treatment of IS in a comprehensive and systematic way by network pharmacology. Methods and Materials. ADME (absorbed, distributed, metabolized, and excreted) is an important property for screening-related compounds in AT, which were screening out of TCMSP, TCMID, Chemistry Database, and literature from CNKI. Then, these targets related to screened compounds were predicted via Swiss Targets, when AT-related targets database was established. The gene targets related to IS were collected from DisGeNET and GeneCards. IS-AT is a common protein interactive network established by STRING Database. Gene Ontology (GO) and Kyoto Encyclopedia of Genes and Genomes (KEGG) pathway enrichment were analysed by IS-AT common target genes. Cytoscape software was used to establish a visualized network for active compounds-core targets and core target proteins-proteins interactive network. Furthermore, we drew a signal pathway picture about its effect to reveal the basic mechanism of AT against IS systematically. Results. There were 53 active compounds screened from AT, inferring the main therapeutic substances as follows: bisasaricin, 3-cyclohexene-1-methanol- $\alpha, \alpha, 4$-trimethyl,acetate, cis,cis,cis-7,10,13-hexadecatrienal, hydroxyacoronene, nerolidol, galgravin, veraguensin, $2^{\prime}$-o-methyl isoliquiritigenin, gammaasarone, and alpha-asarone. We obtained 398 related targets, 63 of which were the same as the IS-related genes from targets prediction. Except for GRM2, remaining 62 target genes have an interactive relation, respectively. The top 10 degree core target genes were IL6, TNF, IL1B, TLR4, NOS3, MAPK1, PTGS2, VEGFA, JUN, and MMP9. There were more than 20 terms of biological process, 7 terms of cellular components, and 14 terms of molecular function through GO enrichment analysis and 13 terms of signal pathway from KEGG enrichment analysis based on $P<0.05$. Conclusion. AT had a therapeutic effect for ischemic via multicomponent, multitarget, and multisignal pathway, which provided a novel research aspect for AT against IS. 


\section{Introduction}

13.7 million people suffered from stroke every year in the world, the second largest reason causing death with around 5.5 million dead $[1,2]$. It not only killed and enabled the elderly people [3] but also made a negative effect for the youth that morbidity rate and incidence had enhanced among them. Especially, mortality rate among young people suffering from stroke in developing countries was in a uptendency, which highly increased disease burden [4]. Over past 30 years, the same situation happened in China [5], where ischemic stroke is the major type of stroke, accounting for $70.8 \%$ [6]. The ratio of mortality rate to disablility rate among the youth in China was $34.5 \%$ to $37.1 \%[7,8]$. Thrombolytic therapy is a common and important method to battle against ischemic stroke. The major mechanism of thrombolytic therapy is that profibrolysin is dissociated with plasmin, which can be used to degrade fibrin to dissolve thrombus. Unluckily, its application is limited and is only applied within 4.5-6 hours after suffering from it. Even if patients could be saved in time via thrombolytic therapy, there were only one-third to half of them accomplishing ischemic reperfusion [9], which might probably lead to transfer hemorrhagic stroke as a side effect.

Traditional Chinese medicine is always used without side effect to reduce disability rates, improve the quality of life, and decrease the risk of recrudesce for cerebral ischemic patients. The major mechanism involved anti-inflammatory reaction, antioxidant, inhibiting neuron apoptosis, promoting vascular regeneration, and maintaining the $\mathrm{BBB}$ permeability. AT was firstly recorded in Sheng Nong's herbal classic thousands years ago, and its dried rhizomes attributed to Araceae Juss, with the effect of reducing phlegm by resuscitation, improving intelligence, dehumidifying, and appetizing [10], which was used to treat tinnitus and epicophosis, Alzheimer disease, epilepsy, depression, and stroke. AT is one important component of Ditan decoction, widely used in clinical stroke treatment, which could decrease the level of inflammatory factors and oxidative stress after cerebral ischemic and inhibit the formation of thrombus to promote stroke patients recovery [11]. Many studies found that AT and its main ingredients had a positive effect on reducing the cerebral ischemic volume [12, 13], which was related to antioxidants [14], and regulating autophagy level $[13,15]$.

It is unclear whether AT and AT-contained ingredients have other mechanisms against IS. Due to lack of systematic and comprehensive explanation about it, network pharmacology was used to elucidate the interactive relation among drug, targets, and disease. Potential target prediction helps us to understand more mechanisms for multiple pharmacological functions of AT and its role in biological network, which will be beneficial for the curative effect improvement $[16,17]$. Many kinds of complex compounds are involved in Chinese medicine, so we applied the network pharmacology method to reveal the effect of TCM. Thus, the aim of this paper is to find out the mechanism of AT against IS in a comprehensive and systemic way.

\section{Methods and Materials}

The flowchart of this study is shown in Figure 1.

2.1. Active Compound Screening and Target Prediction. We searched active compounds AT contained via TCMSP Database [18] (version2.3, http://tcmspw.com/tcmsp.php), TCMID Database (v2.01, http://119.3.41.228:8000/tcmid/ search/), Chemistry Database [19] (http://chemdb.sgst.cn/ scdb/default.asp), and literature from CNKI to provide more useful and important compounds. Based on pharmacokinetics (absorbed, distributed, metabolized, and excreted), we set up a series of parameters, such as $\mathrm{BBB}$ absorption>-0.3 and $\mathrm{DL} \geq 0.18$ [20], to screen suitable compounds in TCMSP Database. Compounds could be obtained from TCMID Database and Chemistry Database without ADME parameters, so we need to get chemical formula of them from PubChem (https://pubchem.ncbi.nlm.nih.gov/) to finish Swiss ADME prediction [21], which was requested that BBB permeability was equal to YES and at least two terms of Drug-likeness were yes. All compounds were screened as above-needed target prediction through Swiss Target [22]. Probability is used to balance the connection between compounds and targets, which is more close to 1 , and it is more connective. We screened targets by a median of probability to establish potential target database related to compounds in AT.

2.2. Obtaining Gene Targets Related to IS. We obtained ISrelated gene targets by searching in Genecards (version 4.14, https://www.genecards.org/) and DisGeNET [23-25] (version 7.0, https://www.disgenet.org/) with the keywords "ischemic stroke," "cerebral ischemic," "cerebral infarction." These gene targets were put into IS-related targets database, respectively. Genes from Genecards in searching different keywords will be screened by its scores, which more than its median are constituted in IS-related targets database from Genecards.

\subsection{AT-Compounds-Targets Network Construction.} AT-compounds-targets visualized network was conducted by Cytoscape (version 3.7.2), where diversity of shape nodes was distinguished from AT, active compounds in AT, and targets, where edges represented the interactive relationship between AT and compounds and compounds and targets.

2.4. Extracting Core Target-Related AT-IS. By the aid of Microsoft Excel, we intersected potential target database related to compounds in AT- and IS-related target database to get the core target related to AT-IS, which was used to draw a Venn diagram online (http://www.bioinformatics. com.cn/).

2.5. Core Targets PPI Network Construction. Core targets related to AT-IS were imported into STRING (version 11.0, https://string-db.org/), selecting species "Homo sapiens". Potential protein targets with a medium confidence score of 


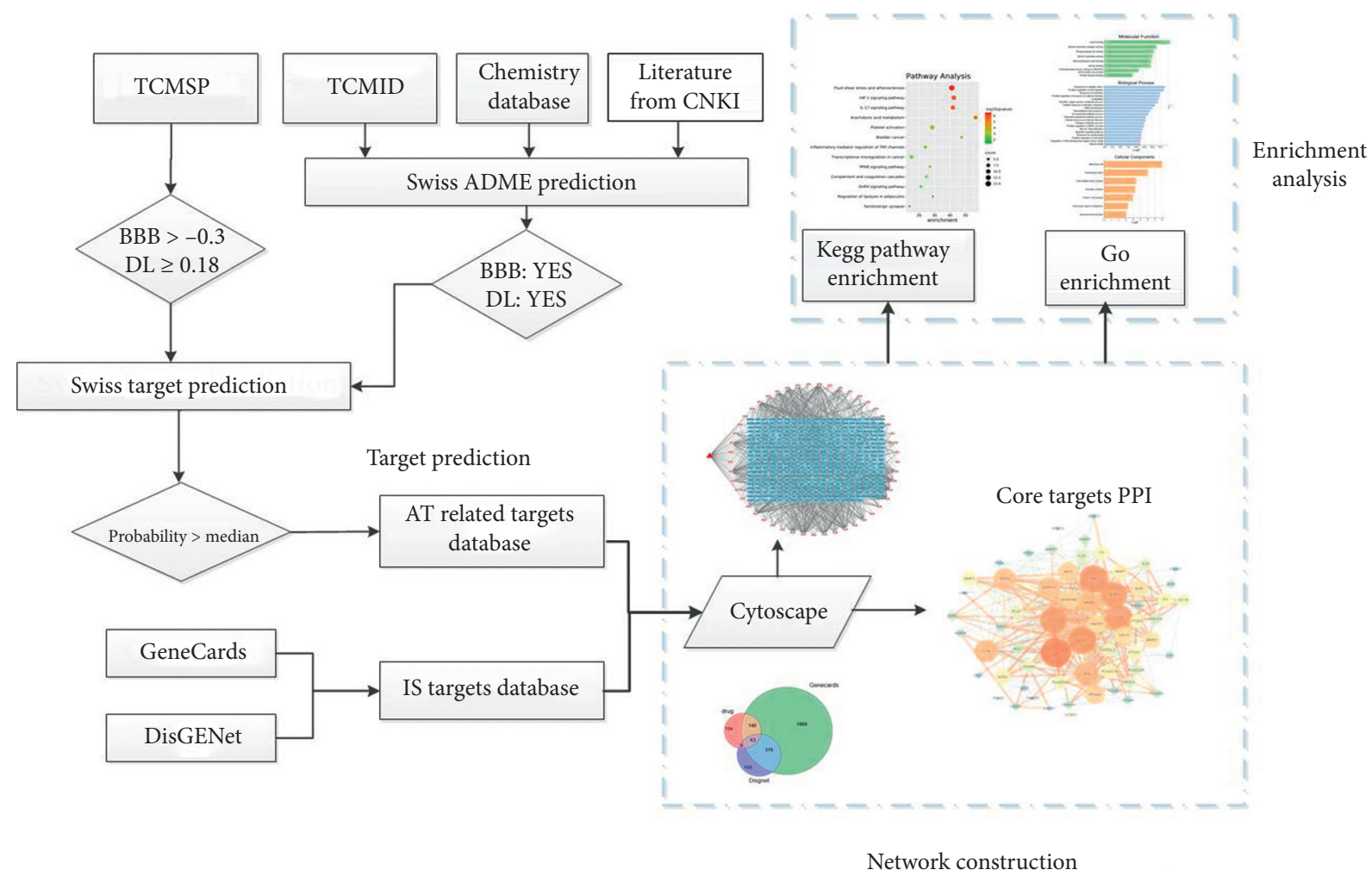

FIgURE 1: Framework for AT against IS based on a network pharmacology.

0.400 were designed as an interaction network, with hiding disconnect core targets to get PPI analysis results. All these results could be used in constructing AT-IS PPI visualized network, based on its degree value by analysing.

2.6. GO and KEGG Enrichment Analysis. GO and KEGG enrichment analysis of core targets related to AT-IS was accomplished by an online tool, Metascape [26] (https:// metascape.org/), choosing species "Homo sapiens" and then clicking "custom analyse." GO enrichment analysis is composed of biological process, molecular function, and cellular components. KEGG enrichment analysis is used to uncover the signal pathway showing potential therapeutic effect of some diseases. Before GO and KEGG enrichment for above core genes, "Min Overlap $=3$," " $P$ value cutoff $=0.01$," and "Min enrichment $=1.5$ " should be set for passway and process enrichment and "Min Network Size $=3$ " and "Max Network Size $=500$ " for protein-protein interaction enrichment.

\section{Results}

3.1. Active Compounds in AT. There were 53 related active compounds satisfying ADME conditions, when filtering out repeat ones. MolID, molecular name, and Pubchem CID of AT active compounds are shown in Table 1. Structure, BBB permeability, and DL of these compounds are filled in Supplementary 3.
3.2. Compound Target Prediction Results and Its Network Construction. Probability scores were used to predict these compound targets, which median is 0.0604024588 (AT-14 (palmitic acid) and AT-22 (2-furfuraldehyde) were lower than this median; no target was related to AT-28 blumenol A; thus, these three compounds were out of the network). 1409 targets were predicted, 398 targets remained after deleting repeat ones, and specific information is shown in Supplementary Table S1. Compound-target network is presented in Figure 2. The network property of compounds in AT, degree, betweenness centrality, closeness centrality, and neighborhood connectivity is involved in Table 2. Nodes with highest connectivity have a high influence on the network [29]. We proposed that the higher the degree it had, the more significant the effect it exerted in therapy. The top 10 degree compounds were bisasaricin, 3-cyclohexene-1-methanol- $\alpha, \alpha, 4$-trimethyl,acetate, cis,cis,cis-7,10,13-hexadecatrienal, hydroxyacoronene, nerolidol, galgravin, veraguensin, $2^{\prime}$-o-methyl isoliquiritigenin, gamma-asarone, and alpha-asarone.

\subsection{AT against IS Core Targets Protein-Protein Interaction} (PPI) Construction. We screened 2391 gene targets in Genecards Database, after filtering out based on median, when 560 gene targets were obtained from DisGeNET (Supplementary Table S2). Genecards Database was established based on gene-centric data from approximately 150 web sources, involved in genomic, transcriptomic, proteomic, genetic, clinical, and functional information. DisGeNET collected data from expert curated repositories, 
TABle 1: Compounds contained in AT.

\begin{tabular}{|c|c|c|c|c|}
\hline No. & MolID & Molecular name & $\begin{array}{l}\text { PubChem } \\
\text { CID }\end{array}$ & Resource \\
\hline 1 & AT-1 & Linalool & 6549 & {$[27]$} \\
\hline 2 & $\mathrm{AT}-2$ & (+)-Longicyclene & 564934 & {$[27]$} \\
\hline 3 & AT-3 & ( \pm )-Camphor & 159055 & {$[27]$} \\
\hline 4 & $\mathrm{AT}-4$ & Bornyl acetate & 6448 & [27] \\
\hline 5 & AT-5 & (-)-Terpinen-4-ol & 5325830 & [27] \\
\hline 6 & AT-6 & Borneol & 64685 & [27] \\
\hline 7 & AT-7 & Shyobunone & 5321293 & [27] \\
\hline 8 & AT-8 & Methyl eugenol & 7127 & [27] \\
\hline 9 & AT-9 & $(+)$-Ledol & 92812 & [27] \\
\hline 10 & AT-10 & (E)-Methyl isoeugenol & 1549045 & {$[27]$} \\
\hline 11 & AT-11 & Elemicin & 10248 & [27] \\
\hline 12 & AT-12 & Alpha-asarone & 636822 & {$[27]$} \\
\hline 13 & AT-13 & $\beta$-Asarone & 5281758 & [27] \\
\hline 14 & AT-14 & Palmitic acid & 985 & {$[27]$} \\
\hline 15 & AT-16 & Galgravin & 101749 & {$[28]$} \\
\hline 16 & AT-17 & $\left(7 \mathrm{~S}, 7^{\prime} \mathrm{S}, 8 \mathrm{R}, 8^{\prime} \mathrm{R}\right)$-Eudesmin & 284823 & {$[28]$} \\
\hline 17 & AT-18 & 1,1,7-Trimethyl-4-methene-decahydro-1H-cycloprop[e]azulene & 91753508 & TCMID \\
\hline 18 & AT-19 & Gamma-asarone & 636750 & TCMID \\
\hline 19 & AT-20 & $\begin{array}{c}\text { 1H-Cyclopropa [a] naphthalene,1a,2,3,5,6,7,7a,7b-octahydro-1,1,7,7a-tetramethyl,[1aR-(1a. } \\
\text { Alpha., 7. Alpha., 7b. Alpha.)] }\end{array}$ & 71717616 & TCMID \\
\hline 20 & AT-21 & $2^{\prime}$-o-Methyl isoliquiritigenin & 5319688 & TCMID \\
\hline 21 & AT-22 & 2-Furfuraldehyde & 7362 & TCMID \\
\hline 22 & AT-23 & Beta-eudesmol-cis-epimer & 12309818 & TCMID \\
\hline 23 & AT-24 & 3,11-Eudesmadien-2-one & 565648 & TCMID \\
\hline 24 & AT-25 & 3-Cyclohexene-1-methanol- $\alpha, \alpha, 4$-trimethyl,acetate & 11736872 & TCMID \\
\hline 25 & AT-27 & Asaronaldehyde & 20525 & TCMID \\
\hline 26 & AT-28 & Blumenol A & 5280462 & TCMID \\
\hline 27 & AT-29 & Carotol & 6432448 & TCMID \\
\hline 28 & AT-30 & Caryophyllene epoxid & 14350 & TCMID \\
\hline 29 & AT-31 & cis,cis,cis-7,10,13-Hexadecatrienal & 5367366 & TCMID \\
\hline 30 & AT-32 & cis-1 $1^{\prime}, 2^{\prime}$-Epoxyasarone & 101009521 & TCMID \\
\hline 31 & AT-33 & Endo-borneol & 1201518 & TCMID \\
\hline 32 & AT-34 & Paeonol & 11092 & TCMID \\
\hline 33 & AT-35 & Eugenol & 3314 & TCMID \\
\hline 34 & AT-37 & Himbaccol & 6432543 & TCMID \\
\hline 35 & AT-38 & Hydroxyacoronene & 102585857 & TCMID \\
\hline 36 & AT-39 & Isoacoramone & 700861 & TCMID \\
\hline 37 & AT-40 & Isolongifolen-5-one & 600416 & TCMID \\
\hline 38 & AT-42 & Naphthalene,1,2,3,4-tetrahydro-1,5,7-trimethyl & 529436 & TCMID \\
\hline 39 & AT-45 & Octenol & 5352836 & TCMID \\
\hline 40 & AT-46 & Phenol,2-methoxy & 460 & TCMID \\
\hline 41 & AT-47 & Terpinen-4-ol- & 20398672 & TCMID \\
\hline 42 & AT -48 & Thymol & 6989 & TCMID \\
\hline 43 & AT-49 & Methylisoeugenol & 637776 & [19] \\
\hline 44 & AT-50 & Graminone & 36679 & [19] \\
\hline 45 & AT-51 & $\alpha$-Cubebene & 442359 & [19] \\
\hline 46 & AT-52 & $\beta$-Cubebene & 93081 & [19] \\
\hline 47 & AT-53 & Nerolidol & 5284507 & [19] \\
\hline 48 & AT-54 & Guaiol & 227829 & [19] \\
\hline 49 & AT-55 & Bisasaricin & 126324 & [19] \\
\hline 50 & AT-56 & Veraguensin & 443026 & [18] \\
\hline 51 & AT-57 & Lupeol & 259846 & [18] \\
\hline 52 & AT-58 & Cycloartenol & 92110 & {$[18]$} \\
\hline 53 & AT-59 & Asatone & 10983193 & {$[18]$} \\
\hline
\end{tabular}

GWAS catalogues, animal models, and the scientific literature, which were identified well in relationship with disease. To avoid data redundancy, genes from Genecards and
DisGeNET were intersected to improve targets relativity. In sum, 63 core gene targets remained after intersection with AT (Figure 3). 


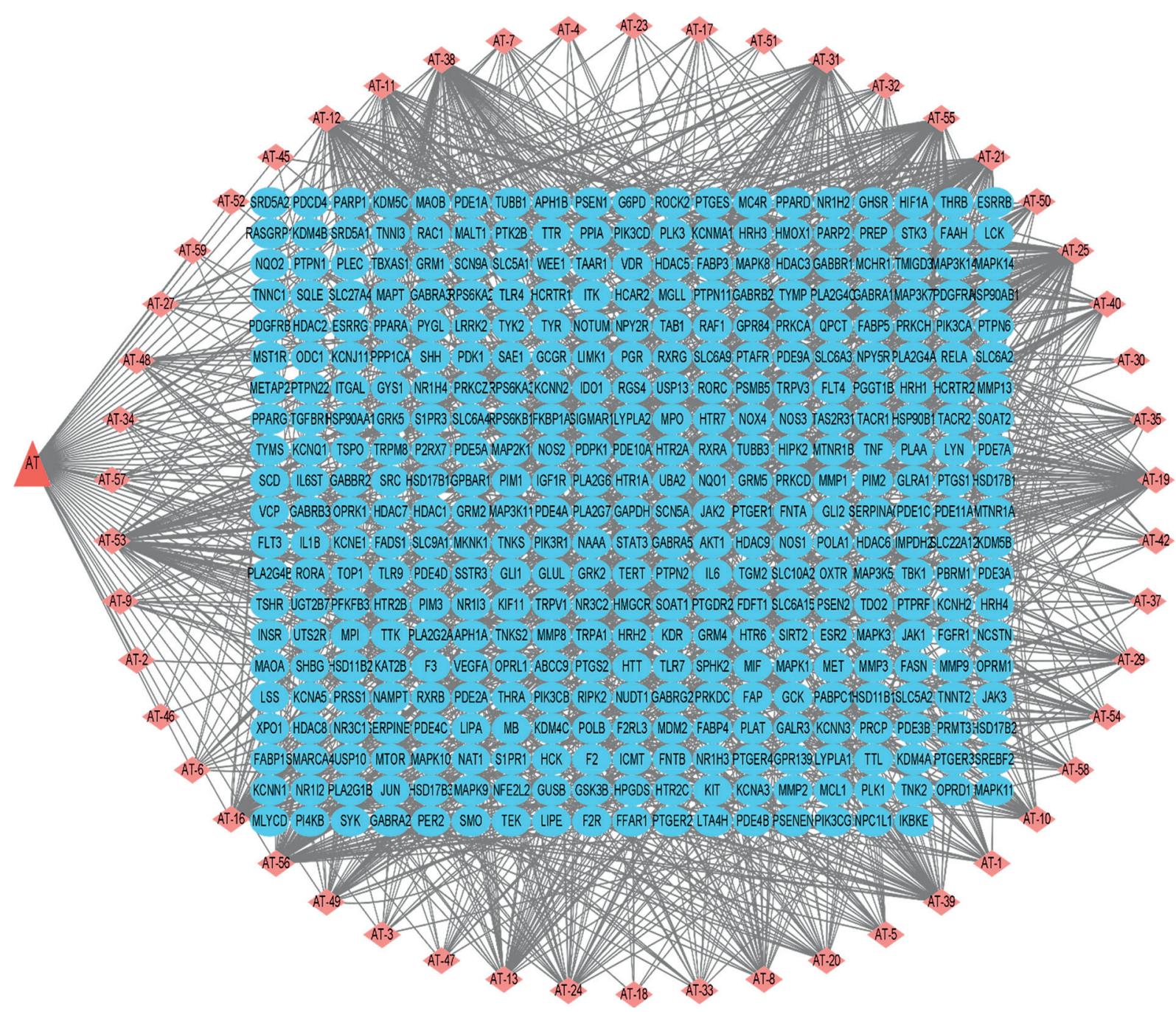

Figure 2: Drug-compounds-targets interaction network (there were 448 nodes and 1459 edges in this network, blue oval nodes represent genes, pink diamonds represent compounds contained in AT, and the red triangle represents AT).

Target GRM2 was dissociated from the PPI network, without interactive relationship, which should be omitted from it. Thus, 62 remaining targets are presented in Table 3 , which were used to construct the PPI visualized network in Figure 4. The lighter color and the bigger size in nodes represented the higher degree value, which made a great contribution to its effect against IS.

3.4. GO Enrichment Analysis. GO analysis of 62 potential core targets for AT against IS was performed by using the Metascape database to understand the relationship between functional units and their underlying significance in the biological system networks. The results were divided into three parts: biological processes (Figure 5(a)), cellular component (Figure 5(b)), and molecular function (Figure 5(c)), which were shown based on $P<0.05$ in statistics.

Firstly, the top 20 terms of biological process for enrichment analysis are shown in Figure 5(a), such as response to oxidative stress, postive regulation of cell migration, response to bacterium, positive regulation of response to external stimulus, coagulation, reactive oxygen species metabolic process, cellular response to nitrogen compound, fatty acid transport, neuroinflammatory response, icosanoid biosynthetic process, organophosphate biosynthetic process, cellular response to external stimulus, collagen metabolic process, positive regulation of cell death, regulation of DNAbinding transcription factor activity, and neuron death. Secondly, there were only 7 terms of cellular components enrichment analysis shown in Figure 5(c) containing membrane raft, extracellular matrix, transcription factor complex, receptor complex, ficolin-1-rich granule, perinuclear region of cytoplasm, and neuromuscular junction. Finally, 14 terms of molecular function are presented in Figure 5(b), which were lipid binding, steroid hormone receptor activity, phospholipase A2 activity, serine hydrolase activity, monocarboxylic acid binding, heme binding, oxidoreductase activity acting on NADPH, protein kinase binding, receptor regulator activity, lipopolysaccharide binding, steroid binding, phosphatase binding, protein kinase activity, and hsp90 protein bind. 
Table 2: Property of compounds in AT.

\begin{tabular}{|c|c|c|c|c|c|}
\hline No. & Molid & Betweenness centrality & Closeness centrality & Degree & Neighborhood connectivity \\
\hline 1 & AT-55 & 0.132711 & 0.389373 & 80 & 5.319444 \\
\hline 2 & AT-25 & 0.128075 & 0.393486 & 78 & 6.102564 \\
\hline 3 & AT-31 & 0.125837 & 0.390734 & 74 & 5.716216 \\
\hline 4 & AT-38 & 0.093817 & 0.390052 & 73 & 7.191781 \\
\hline 5 & AT-53 & 0.097086 & 0.384682 & 67 & 6.030769 \\
\hline 6 & AT-16 & 0.071401 & 0.382705 & 66 & 6.322581 \\
\hline 7 & AT-56 & 0.07275 & 0.382705 & 66 & 6.225806 \\
\hline 8 & AT-21 & 0.141358 & 0.384021 & 64 & 4.390625 \\
\hline 9 & AT-19 & 0.044583 & 0.378173 & 61 & 8.690909 \\
\hline 10 & AT-12 & 0.041561 & 0.376897 & 59 & 8.471698 \\
\hline 11 & AT-39 & 0.098263 & 0.378173 & 55 & 5.690909 \\
\hline 12 & AT-11 & 0.04764 & 0.373746 & 48 & 8.3125 \\
\hline 13 & AT-13 & 0.03325 & 0.373122 & 47 & 8.978723 \\
\hline 14 & AT-24 & 0.023989 & 0.370647 & 43 & 9.72093 \\
\hline 15 & AT- 49 & 0.02007 & 0.3646 & 39 & 10.09091 \\
\hline 16 & AT-8 & 0.015872 & 0.364007 & 36 & 10.28125 \\
\hline 17 & AT-10 & 0.029031 & 0.365794 & 35 & 9.428571 \\
\hline 18 & AT-20 & 0.013698 & 0.362237 & 29 & 9.827586 \\
\hline 19 & AT- 40 & 0.01066 & 0.36165 & 28 & 11.21429 \\
\hline 20 & AT-1 & 0.023586 & 0.361066 & 27 & 8.222222 \\
\hline 21 & AT-29 & 0.014423 & 0.361066 & 27 & 8.703704 \\
\hline 22 & AT-54 & 0.013264 & 0.360484 & 26 & 10.46154 \\
\hline 23 & AT-35 & 0.038727 & 0.3576 & 21 & 7.095238 \\
\hline 24 & AT-33 & 0.009883 & 0.357029 & 20 & 11.65 \\
\hline 25 & AT-5 & 0.009883 & 0.357029 & 20 & 11.65 \\
\hline 26 & AT- 48 & 0.018746 & 0.355326 & 19 & 10.64706 \\
\hline 27 & AT-50 & 0.029511 & 0.356459 & 19 & 8.052632 \\
\hline 28 & AT-32 & 0.013807 & 0.355326 & 17 & 11.11765 \\
\hline 29 & AT- 47 & 0.018568 & 0.354762 & 16 & 10.5 \\
\hline 30 & AT-58 & 0.013403 & 0.354762 & 16 & 13.3125 \\
\hline 31 & AT-17 & 0.005603 & 0.3542 & 15 & 10 \\
\hline 32 & AT-6 & 0.007742 & 0.3542 & 15 & 12.73333 \\
\hline 33 & AT-7 & 0.007742 & 0.3542 & 15 & 12.73333 \\
\hline 34 & AT-57 & 0.007296 & 0.353639 & 14 & 13.57143 \\
\hline 35 & AT-3 & 0.003614 & 0.353081 & 13 & 12.23077 \\
\hline 36 & AT-34 & 0.019332 & 0.353081 & 13 & 8.076923 \\
\hline 37 & AT-37 & 0.007211 & 0.352524 & 12 & 14.33333 \\
\hline 38 & AT-9 & 0.003189 & 0.352524 & 12 & 14.83333 \\
\hline 39 & AT-42 & 0.015185 & 0.351969 & 11 & 11.18182 \\
\hline 40 & AT-23 & 0.004943 & 0.351415 & 10 & 16.9 \\
\hline 41 & AT-27 & 0.027215 & 0.351415 & 10 & 7.5 \\
\hline 42 & AT-4 & 0.002483 & 0.350863 & 9 & 13.66667 \\
\hline 43 & AT-18 & $9.74 \mathrm{E}-04$ & 0.349219 & 6 & 19.33333 \\
\hline 44 & AT-30 & 0.004804 & 0.348674 & 5 & 16.8 \\
\hline 45 & AT- 46 & 0.001824 & 0.348674 & 5 & 15 \\
\hline 46 & AT-51 & $4.81 \mathrm{E}-04$ & 0.348674 & 5 & 19.6 \\
\hline 47 & AT- 45 & $3.60 \mathrm{E}-04$ & 0.348131 & 4 & 18.75 \\
\hline 48 & AT-59 & 0.005322 & 0.348131 & 4 & 14.75 \\
\hline 49 & AT-2 & $2.51 \mathrm{E}-04$ & 0.347589 & 3 & 24.66667 \\
\hline 50 & AT-52 & $2.32 \mathrm{E}-05$ & 0.34705 & 2 & 37 \\
\hline
\end{tabular}

3.5. KEGG Enrichment Analysis. There were 13 terms of signal pathway of AT against IS by KEGG enrichment analysis, which were ordered as ascending tendency according to $P$-value $(P<0.05)$ and contained fluid shear stress and atherosclerosis, HIF-1 signaling pathway, IL-17 signaling pathway, arachidonic acid metabolism, platelet activation, bladder cancer, inflammatory mediator regulation of TRP channels, transcriptional misregulation in cancer, PPAR signaling pathway, complement and coagulation cascades, GnRH signaling pathway, regulation of lipolysis in adipocytes, and serotonergic synapse (Figure 6). According to KEGG enrichment analysis results, we made a 


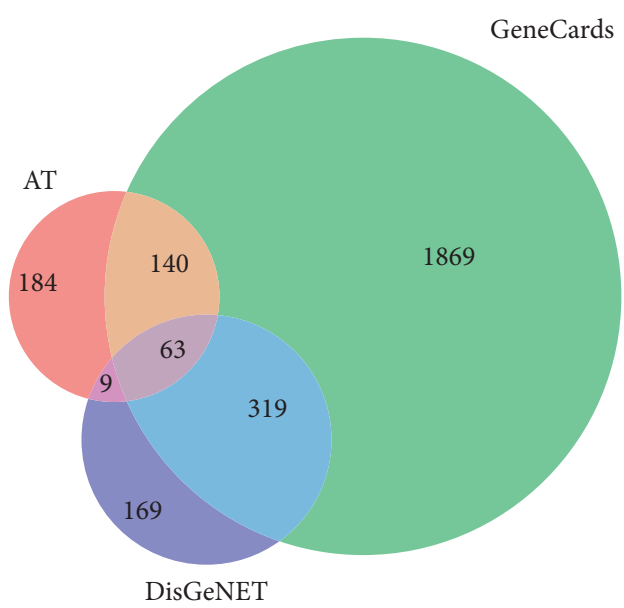

FIGURE 3: Venn diagram: intersection of genes between IS and AT. The part of three circles intersection represented the core target-related IS-AT.

TAble 3: Potential targets related to IS and AT.

\begin{tabular}{|c|c|c|c|c|}
\hline No. & Gene name & Protein name & $\begin{array}{l}\text { UniProt } \\
\text { ID }\end{array}$ & Degree \\
\hline 1 & IL6 & Interleukin-6 & $\mathrm{P} 05231$ & 46 \\
\hline 2 & TNF & TNF-alpha & P01375 & 42 \\
\hline 3 & MAPK1 & MAP kinase ERK2 & P28482 & 41 \\
\hline 4 & PTGS2 & Cyclooxygenase-2 & P35354 & 40 \\
\hline 5 & IL1B & Interleukin-1 beta & P01584 & 39 \\
\hline 6 & VEGFA & Vascular endothelial growth factor A & P15692 & 39 \\
\hline 7 & JUN & Proto-oncogene c-JUN & P05412 & 34 \\
\hline 8 & TLR4 & Toll-like receptor 4 & O00206 & 33 \\
\hline 9 & MMP9 & Matrix metalloproteinase 9 & $\mathrm{P} 14780$ & 31 \\
\hline 10 & NOS3 & Nitric-oxide synthase, endothelial & P29474 & 31 \\
\hline 11 & MAPK14 & MAP kinase p38 alpha & Q16539 & 30 \\
\hline 12 & MPO & Myeloperoxidase & P05164 & 27 \\
\hline 13 & PPARG & Peroxisome proliferator-activated receptor gamma & P37231 & 27 \\
\hline 14 & SERPINE1 & Plasminogen activator inhibitor-1 & P05121 & 25 \\
\hline 15 & MMP2 & Matrix metalloproteinase 2 & P08253 & 23 \\
\hline 16 & HIF1A & Hypoxia-inducible factor 1 alpha & Q16665 & 23 \\
\hline 17 & HMOX1 & HMOX1 & P09601 & 23 \\
\hline 18 & KDR & Vascular endothelial growth factor receptor 2 & P35968 & 23 \\
\hline 19 & MMP1 & Matrix metalloproteinase 1 & P03956 & 20 \\
\hline 20 & NOS2 & Nitric oxide synthase, inducible & P35228 & 20 \\
\hline 21 & MMP3 & Matrix metalloproteinase 3 & P08254 & 19 \\
\hline 22 & PTGS1 & Cyclooxygenase- 1 & P23219 & 18 \\
\hline 23 & PLA2G1B & Phospholipase A2 group 1B & P04054 & 18 \\
\hline 24 & F3 & Coagulation factor VII/tissue factor & P13726 & 18 \\
\hline 25 & F2 & Thrombin & P00734 & 18 \\
\hline 26 & PLA2G4A & Cytosolic phospholipase A2 & P47712 & 16 \\
\hline 27 & PTGES & Prostaglandin E synthase & O14684 & 16 \\
\hline 28 & IGF1R & Insulin-like growth factor I receptor & P08069 & 16 \\
\hline 29 & NFE2L2 & Nuclear factor erythroid 2-related factor 2 & Q16236 & 16 \\
\hline 30 & PPARA & Peroxisome proliferator-activated receptor alpha & Q07869 & 15 \\
\hline 31 & PLAT & Tissue-type plasminogen activator & P00750 & 15 \\
\hline 32 & TLR7 & Toll-like receptor (TLR7/TLR9) & Q9NYK1 & 14 \\
\hline 33 & $\mathrm{NR} 3 \mathrm{C} 1$ & Glucocorticoid receptor & P04150 & 14 \\
\hline 34 & F2R & Proteinase-activated receptor 1 & P25116 & 14 \\
\hline 35 & PLA2G2A & Phospholipase A2 group IIA & P14555 & 13 \\
\hline 36 & NQO1 & Quinone reductase 1 & P15559 & 13 \\
\hline 37 & PIK3CA & PI3-Kinase p110-alpha subunit & P42336 & 13 \\
\hline
\end{tabular}


TABLE 3: Continued.

\begin{tabular}{|c|c|c|c|c|}
\hline No. & Gene name & Protein name & $\begin{array}{l}\text { UniProt } \\
\text { ID }\end{array}$ & Degree \\
\hline 38 & PARP1 & Poly[ADP-ribose]polymerase-1 & P09874 & 11 \\
\hline 39 & NAMPT & Nicotinamide phosphoribosyltransferase & P43490 & 11 \\
\hline 40 & ESR2 & Estrogen receptor beta & Q92731 & 10 \\
\hline 41 & SHH & Sonic hedgehog protein (by homology) & Q15465 & 9 \\
\hline 42 & PLA2G6 & Calcium-independent phospholipase A2 & O60733 & 9 \\
\hline 43 & RAC1 & Ras-related C3 botulinum toxin substrate 1 & P63000 & 9 \\
\hline 44 & FABP4 & Fatty acid binding protein adipocyte & P15090 & 9 \\
\hline 45 & VDR & Vitamin D receptor & P11473 & 8 \\
\hline 46 & LTA4H & Leukotriene A4 hydrolase & P09960 & 8 \\
\hline 47 & TBXAS1 & Thromboxane-A synthase & P24557 & 8 \\
\hline 48 & PLA2G7 & LDL-associated phospholipase A2 & Q13093 & 8 \\
\hline 49 & P2RX7 & P2X purinoceptor 7 & Q99572 & 7 \\
\hline 50 & PIK3CG & PI3-Kinase p110-gamma subunit & P48736 & 7 \\
\hline 51 & FABP1 & Fatty acid-binding protein, liver (by homology) & P07148 & 7 \\
\hline 52 & G6PD & Glucose-6-phosphate 1-dehydrogenase & P11413 & 6 \\
\hline 53 & ITGAL & Leukocyte adhesion glycoprotein LFA-1 alpha & P20701 & 6 \\
\hline 54 & ODC1 & Ornithine decarboxylase & P11926 & 5 \\
\hline 55 & PSEN1 & $\begin{array}{l}\text { Presenilin-1 (PS-1) (EC 3.4.23.-) (protein S182) [cleaved into presenilin-1 NTF subunit; } \\
\text { presenilin-1 CTF subunit; presenilin-1 CTF12 (PS1-CTF12)] }\end{array}$ & P49768 & 4 \\
\hline 56 & $\mathrm{NR} 3 \mathrm{C} 2$ & Mineralocorticoid receptor & P08235 & 4 \\
\hline 57 & FADS1 & Fatty acid desaturase 1 & O60427 & 3 \\
\hline 58 & HDAC9 & Histone deacetylase 9 & Q9UKV0 & 2 \\
\hline 59 & PRKCH & Protein kinase $\mathrm{C}$ eta & P24723 & 2 \\
\hline 60 & LIMK1 & LIM domain kinase 1 & P53667 & 2 \\
\hline 61 & PDE4D & Phosphodiesterase 4D & Q08499 & 1 \\
\hline 62 & KCNQ1 & Voltage-gated potassium channel, IKs; KCNQ1(Kv7.1)/KCNE1(MinK) & P51787 & 1 \\
\hline
\end{tabular}

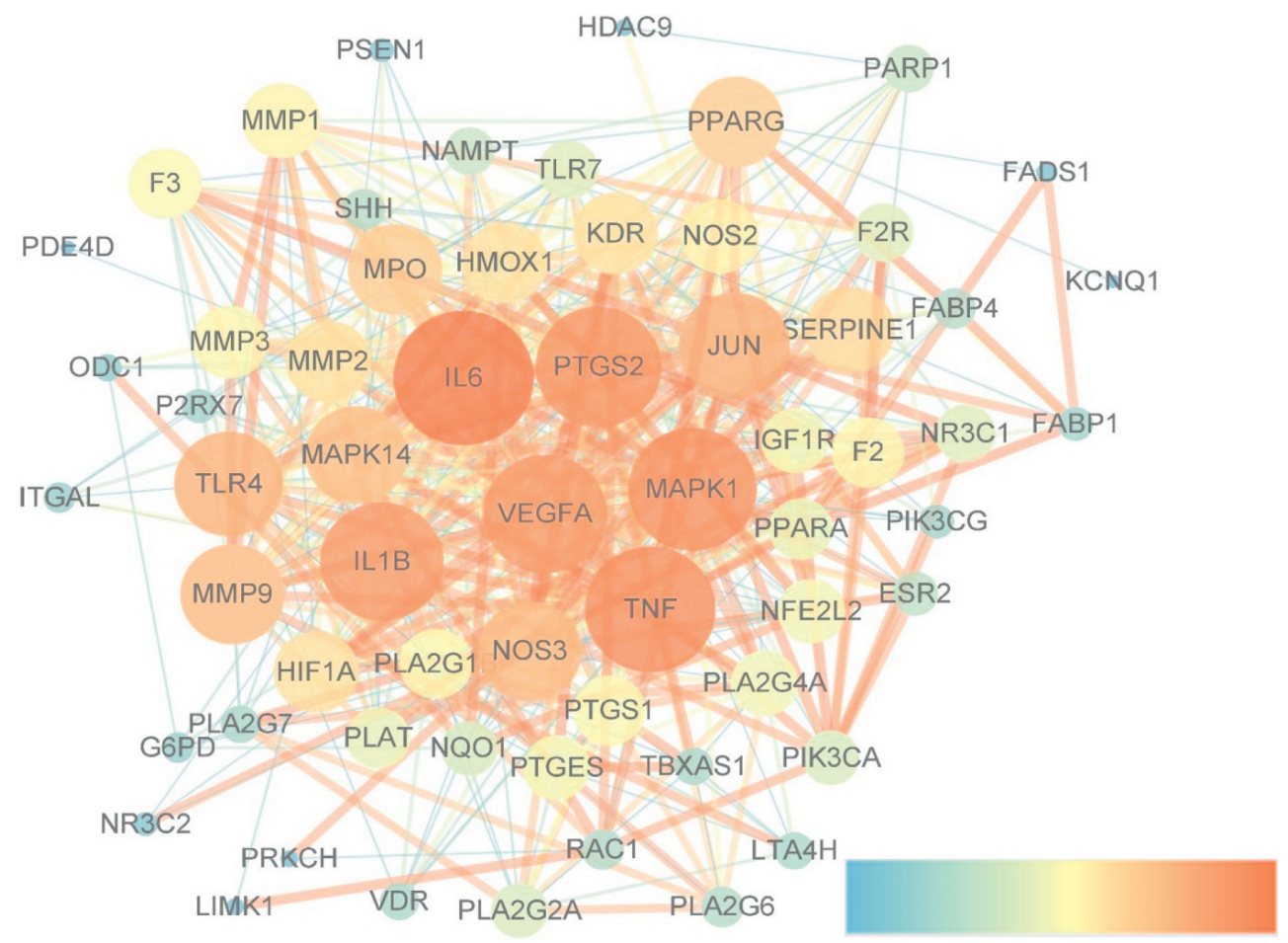

Figure 4: PPI network of potential core targets related to AT and IS. Left to right represented dark to light. The nodes in a lighter color and a bigger size represented a higher degree. The edges in a lighter color and a wider size represented a higher combined score. 


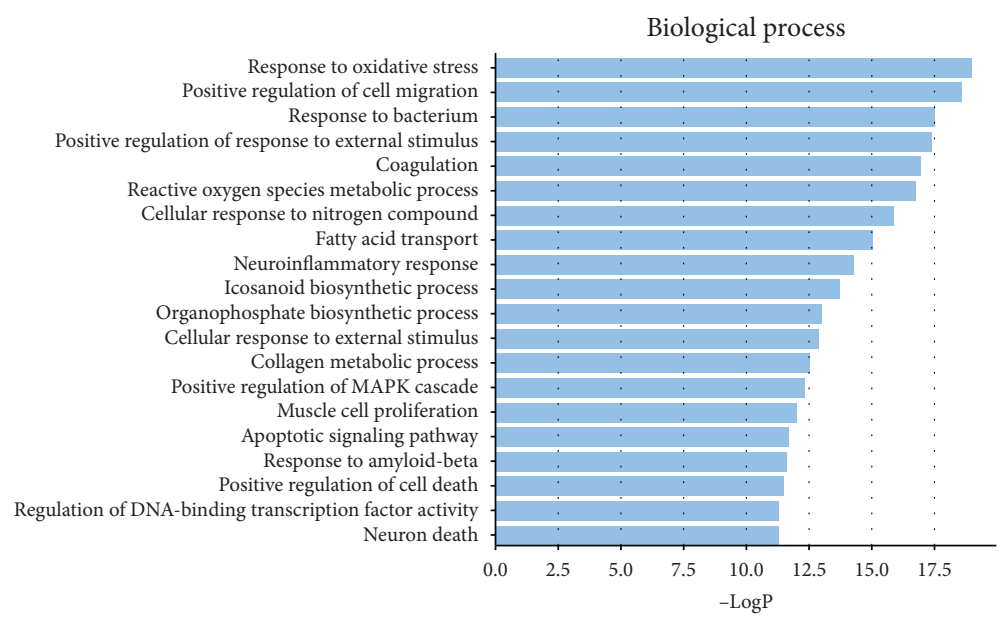

(a)

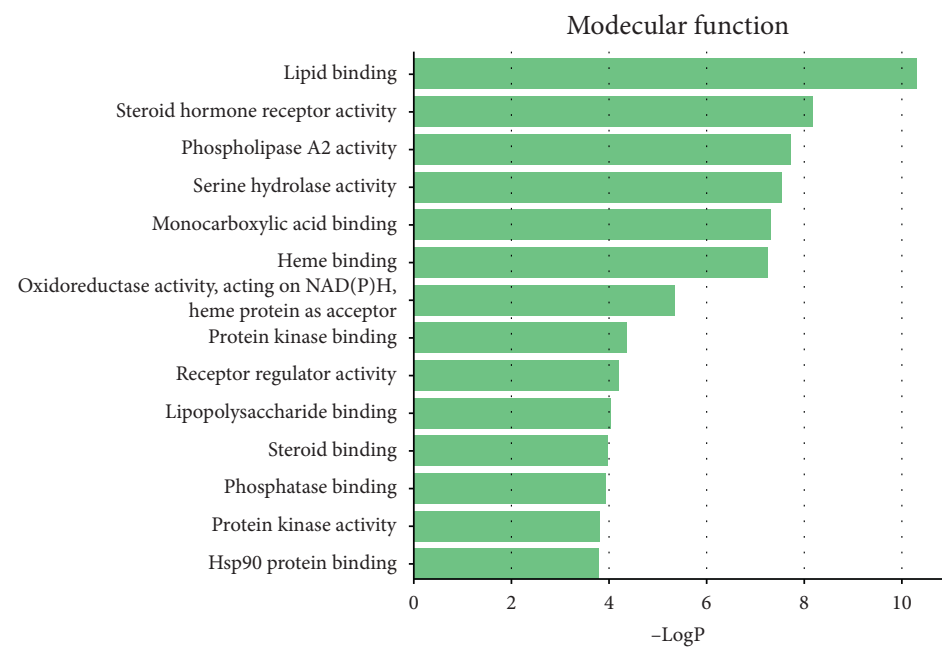

(b)

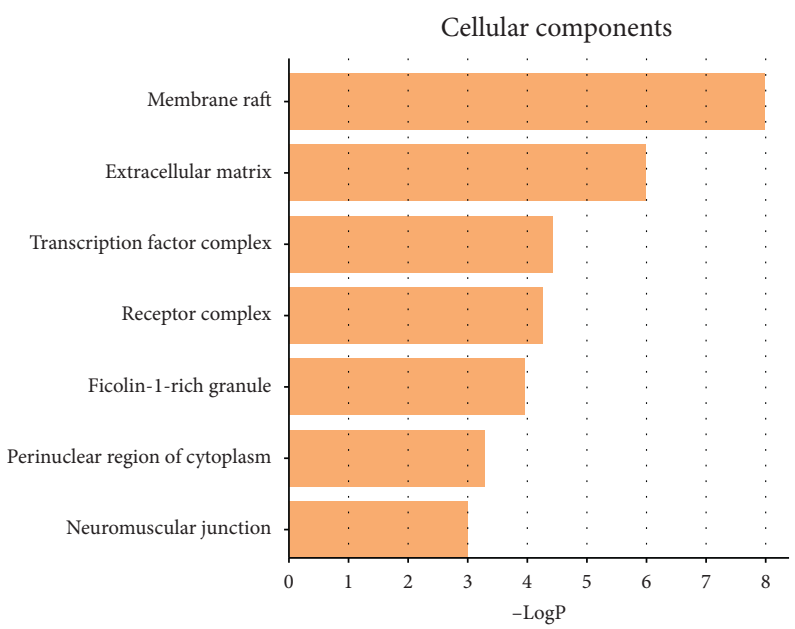

(c)

FIGURE 5: GO enrichment analysis: (a) the top 20 terms of biological process; (b) 14 terms of molecular function; (c) 7 terms of cell components with $P<0.05$. 


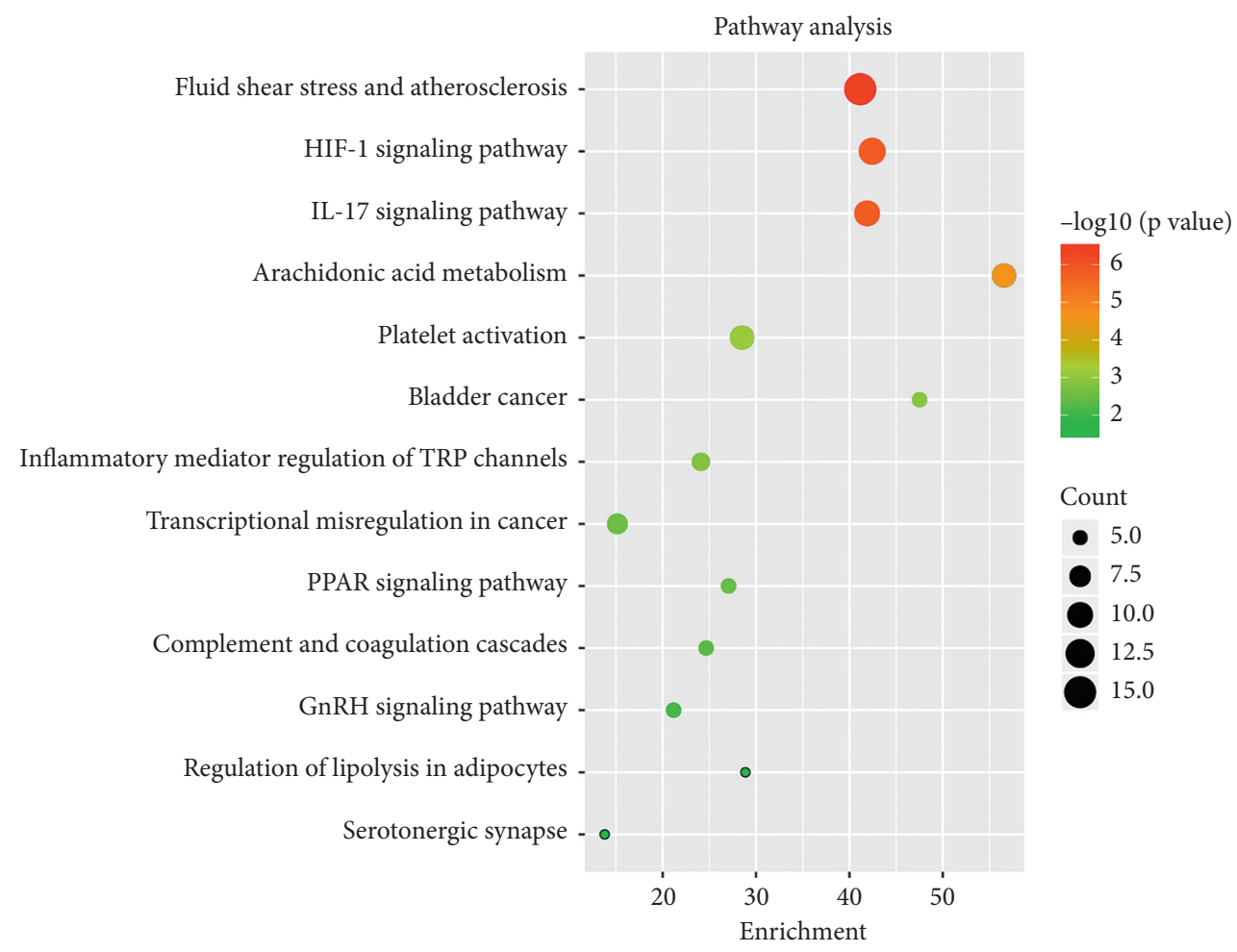

FIGURE 6: KEGG pathway enrichment analysis. "Enrichment" represented the number of target genes belonging to a pathway and the count of the annotated genes located in the pathway. The size of the dot represented the number of core genes related to AT-IS in the pathway and the color of the dot reflected the extent of significance in statistics $(P<0.05)$. Bigger size and lighter color of dot meant a higher level of enrichment.

systematic summary for these core targets in the signal pathway in Figure 7. And we were informed for the specific role of these targets in a certain signal pathway (Figure 8).

\section{Discussion}

Absorbed, distributed, metabolized, and excreted are significant parameters to determine the transformation and delivery of one drug in the body. A CNS-active drug having a high $\mathrm{BBB}$ permeability is crucial for its antistoke effect for entering into the brain. It was evidenced than if BBB permeability was lower than -0.30 , and it had no effect on entering the brain through $\mathrm{BBB}$, so we set it for more than that [20]. Only 6 compounds (bisasaricin, veraguensin, lupeol, cycloartenol, asatone, and eudesmin) fitted this ADME screening in TCMSP. So it was hard to point out AT in treating IS systemically and comprehensively, and we searched on TCMID Database, Chemistry Database, and literature to supply its composition to find more potential targets. In total, we found 50 compounds in AT with effect of treating IS probably, which mainly composed of volatile oil. $\alpha$-Asarone and $\beta$-asarone accounted for $95 \%$ in volatile oil, both of which could promote neuron differentiation via PI3K by aid of growth factors [30]. In our study, it was confirmed that $\alpha$-asarone, with a high degree value in compound-target network (degree $=59$ ), uncovered its core role in AT.

There were 62 core targets for AT against IS, IL6, TNF, IL1B, TLR4, NOS3, MAPK1, PTGS2, VEGFA, JUN, and
MMP9 involved in the top 10 degree targets. IL6, TNF, IL1B, TLR4, and NOS3 are related to the HIF-1 signal pathway, fluid shear stress and atherosclerosis, IL-17 signal pathway, and inflammatory mediator regulation of TRP channels, which are called inflammatory factors as effect for enhancing the level of inflammatory reaction in cerebral ischemic [31-33]. GO enrichment results contained some biological processes related to antistroke closely, such as response to oxidative stress, positive regulation of cell migration, coagulation, reactive oxygen species metabolic process, cellular response to nitrogen compound, fatty acid transport, and neuroinflammatory response. Similarly, some of KEGG enrichment pathways were related to secondary prevention of ischemic stroke by intervening atherosclerosis, regulation of lipolysis in adipocytes, coagulation cascades, arachidonic acid (AA) metabolism, and platelet activation. Others might have a potential therapeutic effect for IS, which were PPAR signal pathway, HIF-1 signal pathway, and inflammatory mediator regulation of TRP channels.

Atherosclerosis is the main pathological mechanism leading to cerebral ischemic, half of which has a connection with it. It was commonly known that it was beneficial to decrease the incidence or palindromia of cerebral ischemic by intervening atherosclerosis as secondary prevention [34]. In fact, fluid shear stress and atherosclerosis is a signal pathway with dual regulation for the formation of atherosclerosis, containing the biological process, antiatherosclerosis [35], and proatherosclerosis $[36,37]$, where nuclear transcripts Nrf2 and 


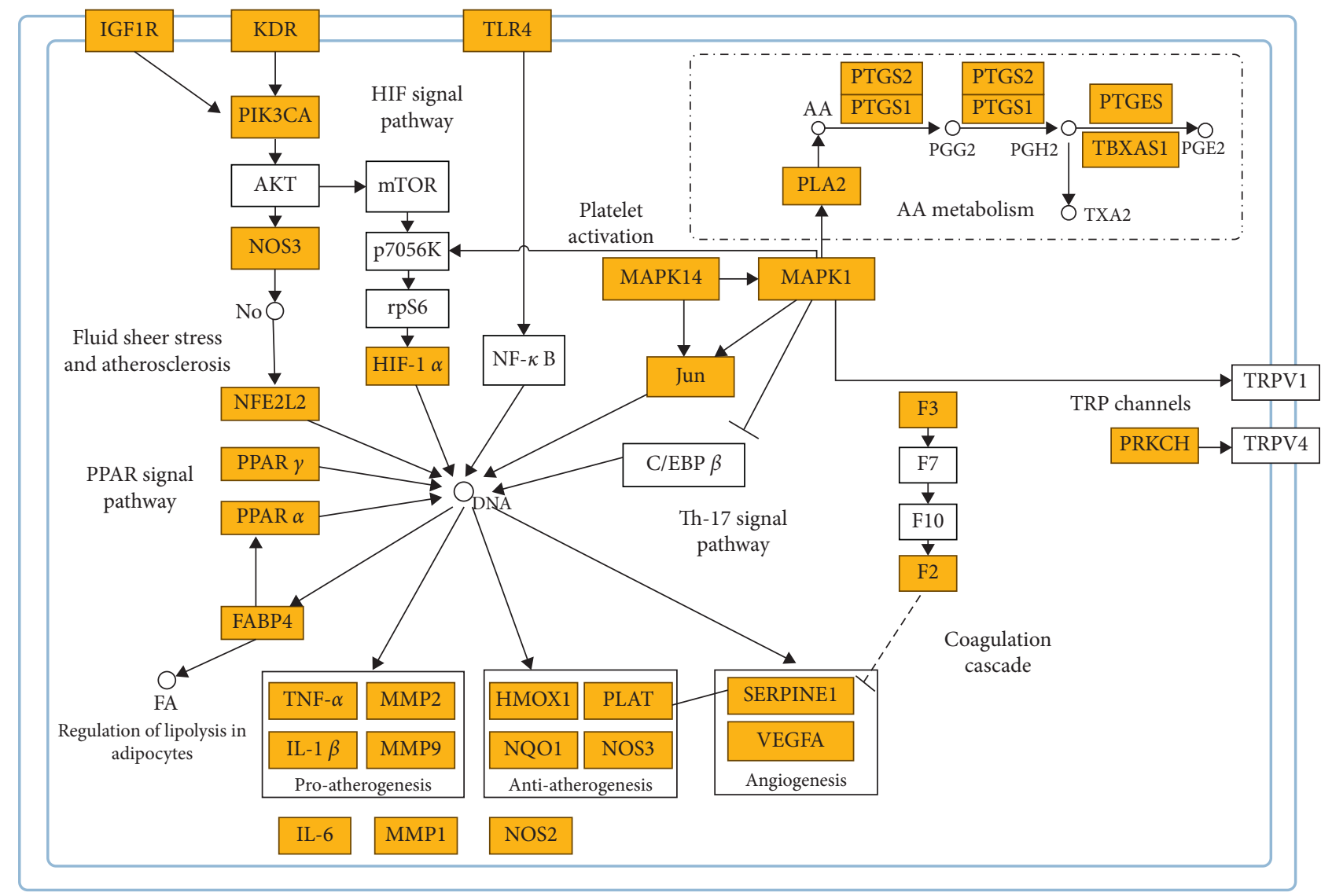

FIGURE 7: Systematic understanding of the antistroke mechanism of AT. The orange nodes represented the targets related to AT and IS and white ones related stroke. AA: arachidonic acid; FA: fatty acid. Full lines represent targets interacting with each other directly, and dotted lines represent indirect interaction.

JUN (a part of AP-1 protein dimers) [38] are responsible for the process, respectively [39]. There was a evidence that lupeol, one component of AT, decreased lipid peroxidation level in the early stage of hypercholesterolemia artery atherosclerosis [40]. It was demonstrated that lupeol could exert a protective effect against cerebral I/R by activating Nrf2 and inhibiting p38MAPK, with decreasing proinflammatory factors TNF- $\alpha$, IL$1 \beta$, and IL-6, increasing anti-inflammatory factor IL-10, and suppressing oxidative stress level [41]. JNK and p38 MAPK promotes the expression of JUN to promote the proatherosclerosis process. Phosphorylation of p38-MAPK and JNK was inhibited by lupeol, when it suppresses the activation of microglia and astrocytes induced by LPS [42]. In addition, another component, bornyl acetate, which was found to promote HUVEC cell vitality recovery, insulted by ox-LDL, suppressing monocytes adhered to HUVEC cells, and decreasing TNF- $\alpha$ and IL- $1 \beta$ proinflammatory factors expression to antiatherosclerosis [43].

Disorder of fatty metabolism not only induces atherosclerosis but also is a high risk of stroke. It is necessary to regulate adipose lipolysis to avoid metabolism syndrome occurrence as possible to decrease the incidence of stroke indirectly. However, it required to further prove the role of adipose lipolysis in ischemic stroke for fat mice. It was confirmed that stroke rats with a high level of inflammatory factor in plasma and adipose tissue induced adipose lipolytic enzymes and free fatty acids expression increased [44]. It is a pity that amounts of compounds were conformed having a positive effect on fatty metabolism not through FABP4. Eudesmin could downregulate S6K1-H2BS36p to impair lipoblast differentiation [45]. Eugenol inhibited hepatic lipid accumulation by downregulating SREBP1 gene expression via increasing CAMKK, AMPK, and acetyl-CoA carboxylase (ACC) and suppressing phosphorylation of mammalian target of rapamycin (mTOR) and p70S6K [46]. Thymol could enhance PPAR $\gamma$ and PPAR $\delta$ expression through overactivated p38MAPK, AMPK, and PKA to promote white adipose cell browning and increased lipid degeneration [47].

PPAR signal pathway has a close relationship with fatty metabolism, which also exerts a protective effect on cerebral ischemic. PPAR $\alpha$ and $\operatorname{PPAR} \gamma$, nuclear transcription from nuclear receptor family, and PPAR $\alpha / \gamma$ agonist decreased inflammatory reaction to induce the neuroprotective effect after cerebral ischemic [48]. $\alpha$-Asarone conjugate structure activated by PPAR $\alpha$, increasing CPT1A gene expression related to degeneration and metabolism of fatty acid [49]. Linalool was identified as a PPAR $\alpha$ ligand directly [50], which improved postischemic neurological scores and cognitive ability by decreasing the COX-2, IL- 6 , Nrf expression in cortex, and hippocampus of IS rat [51]. 


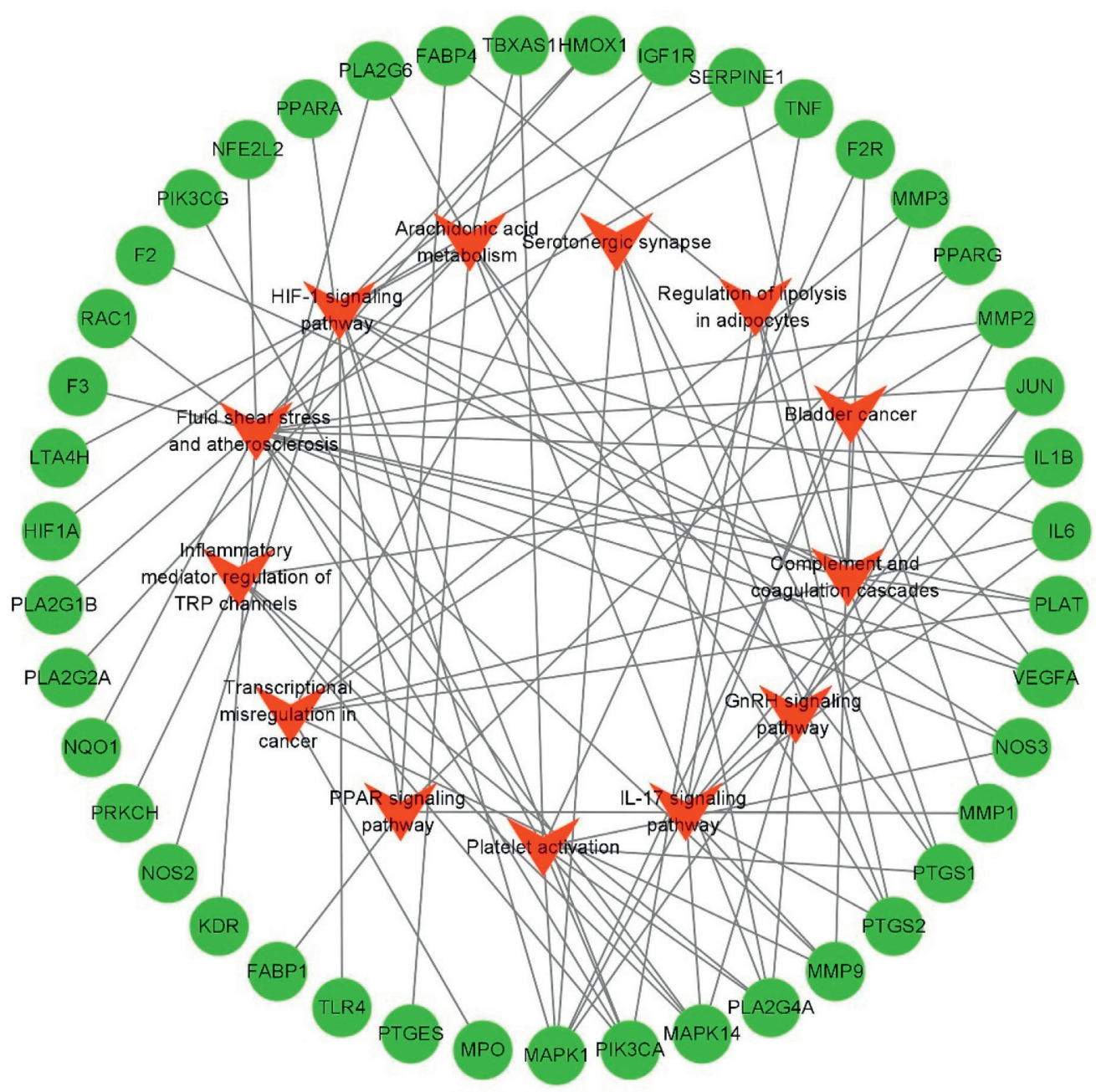

FIGURE 8: The network of potential core targets and signaling pathways in the treatment of IS by AT. Red nodes represent the potential signal pathway for AT against IS, and green nodes represent the potential core targets on it.

Antiplatelet aggregative activity and anticoagulation are common and major therapy to prevent emboli and thrombus formation for ischemic stroke. Previous studies confirmed AT suppressing platelet activation and thrombus formation $[52,53]$. Platelet activation and accumulation is a cascade process, and $\mathrm{TXA}_{2}$ is a positive feedback protein, platelet agonist, a second wave mediater, which is not unnecessary for its accumulation. It can trigger platelet activated by $\mathrm{G}$ proteincoupled receptor [54]. In our study, we found MAPK, ERK, PLA, PTGS1, and TBXAS1 core targets involved in the process of platelet activation to regulate $\mathrm{TXA}_{2}$ produce. SQ29548, $\mathrm{TXA}_{2} \mathrm{R}$ antagonist, was found to inhibit microglia/macrophages activation and enrichment to reduce injury of cerebral ischemic [55]. It has been proved based on network pharmacology that $\beta$-asarone could be against coagulation via APP, PTGS2, and TBXAS1 [56]. Eugenol inhibited platelet activation better than elemicin, in a dose-dependent manner, which also preceded the effect of ASA-COX inhibitors such as asprin [57]. Paeonol increased $\mathrm{NO}$ and $\mathrm{PGI}_{2}$ expression and decreased ET-1 and $\mathrm{TXA}_{2}$ expression to inhibit platelet activation and accumulation to suppress thrombus formation [58]. 3,11-Eudesmadien-2-one [59] and $\alpha$-asarone [53] also had a similar effect, which is indispensable to further identify its specific mechanism.
In addition, arachidonic acid (AA) metabolism promotes platelet accumulation. COX-2(PTGS2) makes AA degeneration, whereas PLA2 promotes AA synthesis, which takes participation in $\mathrm{TXA}_{2}$ formation leading to platelet accumulation. A series of evidence has proved that a high level of COX-2 and PLA2 was filled with MCAO animal models and stroke patients [60-63]. Eugenol inhibited AA metabolism via cyclooxygenase and lipoxygenase pathways in human platelets [64]. In vitro, paeonol inhibited MAPKs activation in macrophages, decreasing iNOS, COX2, and IL6 expression to inhibit inflammatory reaction [65]. $\alpha$-Iso-cubebene inhibited iNOS, COX2, and MMP9 expression and the phosphorylation of JNK and p38 to exert a neuroprotective effect, which was released by microglia amyloid beta induced [66]. However, whether paeonol or $\alpha$-iso-cubebene has a neuroprotective effect for IS via inhibiting AA metabolism need to be further proved.

AT antithrombus was involved in an intrinsic way of coagulation cascades. FXa activated JNK pathway through PAR-1, which triggered F2 to induce neuron death [67]. In vitro, hippocampus clips insulted by OGD strengthened the activity of thrombus, which enhanced 
strength of synapsis by NAMDR; oppositely, if is inhibited thrombin/PAR-1, plasticity of synapsis was recovered [68]. It was a pity that there was no study proving AT or its components against thrombus by an intrinsic way of coagulation cascades so that we inferred it would be a novel research direction for neuroprotection of AT. Because of increasing Th-17 and decreasing Treg contributing to the pathology process of cerebral ischemic in clinic [69], we inferred that another new therapeutic point of view might be related to Th17 differentiation. In vivo, thymol decreased Th1/Treg and Th17/Treg in spleen for mice immune to Ova, which avoided overactivation of Th1 and Th17 [70].

Acute cerebral ischemic patients had a high level of HIF- $1 \alpha$ in the early stage of clinic, indicating a serious expectation for 90 days [71]. TLR4 is one target in the HIF-1 signal pathway, where neutralizing HIF- $1 \alpha$ weakened the increase in TLR4 in BV-2 cells in hypoxia condition with downregulation of TNF- $\alpha$ expression [72]. In fact, paeonol inhibited TLR4 expression, without influence of TNF- $\alpha$ [73]. $\beta$-Asarone had the ability of antioxidation, which decreased HIF- $1 \alpha$ in cortex [74].

Both HRPA1 and TPR1 are members of TRP superfamily, which are structurally dependent nonselective cation channels and mediators of several signaling pathways. Methyl eugenol was proved to be hTPR1 agonist, selectively activating hTPRA1 [75]. Microglia was filled with TPRV1, which was activated to strengthen transmission of glutamate [76]. Previous study was identified that thymol improved spontaneous excitability transmission of spinal substantia gelatinosa neurons [77]. However, it is unknown whether methyl eugenol or thymol has a protective effect for IS via inflammatory mediator regulation of TRP channels, which needs more experiments to be proved. TPRV4 activated prompted expression of VEGFA and eNOS in cerebral ischemic mice, which was beneficial for proliferation and migration of neural stem cells (NPCs) and angiogenesis [78]. TPRV4 agonist impaired cerebral in IS rats by upregulating PI3K/AKT and downregulating p38MAPK (MAPK14) [79].

Taken together, these signal pathways, related to AT preventing and treating IS, could be approximately classified into three categories as follows: atherosclerosis, regulation of lipolysis in adipocytes, and PPAR signal pathway were associated with lipid metabolism; coagulation cascades, arachidonic acid (AA) metabolism, and platelet activation had a connection with the therapy of antiplatelet aggregative activity and anticoagulation; and HIF-1 signal pathway, inflammatory mediator regulation of TRP channels, and Th17 differentiation might be the potential therapeutic signal pathway. In addition, we inferred that an intrinsic way of coagulation cascades and Th17 differentiation was probably new therapeutic or preventive direction for the role of AT against IS.

\section{Conclusion}

In summary, we explored and explained multiple compounds, multiple pathways, and multiple targets of ATregulated ischemic stroke treatment based on a network pharmacology method. Our data indicated that amounts of compounds contained in AT might prevent and treat ischemic stroke through some signal pathways, most of which need an elaborated detailed mechanism in the related signal pathway. Two novel research directions (IL-17 signaling pathway and complement and coagulation cascades) of AT therapeutic for preventing IS were proposed. In the future, more studies should focus on providing experimental evidence and enhancing the effect of AT in the cerebral ischemic on a comprehensive level and improving ability of AT targeting to the brain and crossing the BBB.

\section{Data Availability}

The data used to support the findings of this study are available from the corresponding author upon request.

\section{Conflicts of Interest}

The authors declare that they have no conflicts of interest.

\section{Authors' Contributions}

FZ L conceived and designed the study. FZ L and Q Z wrote the paper. SX L accomplished statistical data. YZ X, DR Z, and $S \mathrm{~W}$ gathered active components and target information. FZ L, LQ Z, and Y G reviewed and edited the manuscript. All authors read and approved the manuscript.

\section{Acknowledgments}

This research was supported by the National Natural Science Foundation of China (Grant no. 81573926) and National Key R\&D Program of China (Grant no. 2018YFC1705000).

\section{Supplementary Materials}

Table S1: compound targets for each component in AT by prediction. Table S2: IS-related targets in GeneCards and DisGeNET. Table S3: specific property of compounds contained in AT. (Supplementary Materials)

\section{References}

[1] GBD 2016 Stroke Collaborators, "Global, regional, and national burden of stroke, 1990-2016: a systematic analysis for the global burden of disease study 2016," Lancet Neurology, vol. 18, no. 5, pp. 439-458, 2019.

[2] M. P. Lindsay, B. Norrving, R. L. Sacco et al., "World stroke organization (WSO): global stroke fact sheet 2019," International Journal of Stroke, vol. 14, no. 8, pp. 806-817, 2019.

[3] C. Mattiuzzi and G. Lippi, "Worldwide disease epidemiology in the older persons," European Geriatric Medicine, vol. 11, no. 1, pp. 147-153, 2020.

[4] R. V. Krishnamurthi, A. E. Moran, V. L. Feigin et al., "Stroke prevalence, mortality and disability-adjusted life years in adults aged 20-64 years in 1990-2013: data from the global burden of disease 2013 study," Neuroepidemiology, vol. 45, no. 3, pp. 190-202, 2015.

[5] W. Wang, B. Jiang, H. Sun et al., "Prevalence, incidence, and mortality of stroke in China," Circulation, vol. 135, no. 8, pp. 759-771, 2017. 
[6] D. Wang, J. Liu, M. Liu, C. Lu, M. Brainin, and J. Zhang, "Patterns of stroke between university hospitals and nonuniversity hospitals in mainland China: prospective multicenter hospital-based registry study," World Neurosurgery, vol. 98, pp. 258-265, 2017.

[7] Z. L. Hao, M Liu, and W. Li, "Basic characteristics and functional outcomes of consecutive 3123 patients in Chengdu stroke registry," Chinese Journal of Neurology, vol. 44, no. 12, pp. 826-831, 2011.

[8] Z. Wang, J. Li, C. Wang et al., "Gender differences in 1-year clinical characteristics and outcomes after stroke: results from the China National Stroke Registry," PLoS One, vol. 8, no. 2, p. e56459, 2013.

[9] "Tissue plasminogen activator for acute ischemic stroke," The New England Journal of Medicine, vol. 333, no. 24, pp. 1581-1587, 1995.

[10] C. P. Commission, Pharmacopoeia of the People's Republic of China, China Medical Science Press, Beijing, China, 2015.

[11] Y. Cao, A. R. Li, and H. Tang, "Effects of ditan decoction on prognosis of patients with cerebral infarction and thromboelastogram index and vascular endothelial factor level," World Chinese Medicine, vol. 15, no. 5, pp. 755-759, 2020.

[12] P. K. Shukla, V. K. Khanna, M. M. Ali, R. Shukla, M. Y. Khan, and R. C. Srimal, "Neuroprotective effect of Acorus calamus against middle cerebral artery occlusion-induced ischaemia in rat," $\mathrm{Hu}$ man \& Experimental Toxicology, vol. 25, no. 4, pp. 187-194, 2006.

[13] X. Sun, D. Wang, T. Zhang et al., "Eugenol attenuates cerebral ischemia-reperfusion injury by enhancing autophagy via AMPK-mTOR-P70S6K pathway," Frontiers in Pharmacology, vol. 11, p. 84, 2020.

[14] J. Bernardo, F. Ferreres, Á. Gil-Izquierdo, P. Valentão, and P. B. Andrade, "Medicinal species as MTDLs: turnera diffusa Willd. Ex Schult inhibits CNS enzymes and delays glutamate excitotoxicity in SH-SY5Y cells via oxidative damage," Food and Chemical Toxicology, vol. 106, pp. 466-476, 2017.

[15] L. Liu, Y.-Q. Fang, Z.-F. Xue, Y.-P. He, R.-M. Fang, and L. Li, "Beta-asarone attenuates ischemia-reperfusion-induced autophagy in rat brains via modulating JNK, p-JNK, Bcl-2 and Beclin 1," European Journal of Pharmacology, vol. 680, no. 1-3, pp. 34-40, 2012.

[16] A. L. Hopkins, "Network pharmacology: the next paradigm in drug discovery," Nature Chemical Biology, vol. 4, no. 11, pp. 682-690, 2008.

[17] S. I. Berger and R. Iyengar, "Network analyses in systems pharmacology," Bioinformatics, vol. 25, no. 19, pp. 2466-2472, 2009.

[18] J. Ru, P. Li, and J. Wang, "TCMSP: a database of systems pharmacology for drug discovery from herbal medicines," Journal of Cheminformation, vol. 6, p. 13, 2014.

[19] Chinese Academy of Sciences, Shanghai Institute of Organic Chemistry, Chemistry Database, Shanghai, China, 2016.

[20] Y. Li, J. Zhang, L. Zhang et al., "Systems pharmacology to decipher the combinational anti-migraine effects of Tianshu formula," Journal of Ethnopharmacology, vol. 174, pp. 45-56, 2015.

[21] A. Daina and V. Zoete, "A boiled-egg to predict gastrointestinal absorption and brain penetration of small molecules," ChemMedChem, vol. 11, no. 11, pp. 1117-1121, 2016.

[22] D. Gfeller, O. Michielin, and V. Zoete, "Shaping the interaction landscape of bioactive molecules," Bioinformatics, vol. 29, no. 23, pp. 3073-3079, 2013.

[23] J. Piñero, J. M. Ramírez-Anguita, J. Saüch-Pitarch et al., "The DisGeNET knowledge platform for disease genomics: 2019 update," Nucleic Acids Research, vol. 48, no. D1, pp. D845-d855, 2020.
[24] J. Piñero, À. Bravo, N. Queralt-Rosinach et al., "DisGeNET: a comprehensive platform integrating information on human disease-associated genes and variants," Nucleic Acids Research, vol. 45, no. D1, pp. D833-d839, 2017.

[25] J. Piñero, N. Queralt-Rosinach, A. Bravo et al., "DisGeNET: a discovery platform for the dynamical exploration of human diseases and their genes," Database, vol. 2015, p. bav028, 2015.

[26] Y. Zhou, B. Zhou, and L. Pache, "Metascape provides a biologistoriented resource for the analysis of systems-level datasets," Nature Communications, vol. 10, no. 1, p. 1523, 2019.

[27] B. Wang, G. Pei, X. L. Wang et al., "Determination of 26 volatile components in Acorus tatarinowii by GC-MS," Lishizhen Medcine and Mate Ria Medica Research, vol. 26, no. 11, pp. 2627-2630, 2015.

[28] G. Ni and D. Q. Yu, "Studies on the chemical constituents of Acorus tatarinowii," China Journal of Chinese Materia Mediac, vol. 38, no. 4, pp. 569-573, 2013.

[29] M. Zhang, J. Deng, and C. V. Fang, Molecular Network Analysis and Applications, John Wiley \& Sons, Hoboken, NJ, USA, 2010.

[30] K. Y. C. Lam, J. Chen, C. T. W. Lam et al., "Asarone from acori tatarinowii rhizoma potentiates the nerve growth factor-induced neuronal differentiation in cultured PC12 cells: a signaling mediated by protein kinase A," PLoS One, vol. 11, no. 9, Article ID e0163337, 2016.

[31] S. M. Hwang, Y. J. Lee, J. J. Yoon et al., "Prunella vulgaris suppresses HG-induced vascular inflammation via Nrf2/HO-1/ eNOS activation," International Journal of Molecular Sciences, vol. 13, no. 1, pp. 1258-1268, 2012.

[32] L. Zheng, Y. Wang, J. Liu et al., "Pro-inflammatory cytokine network in peripheral inflammation response to cerebral ischemia," Neuroscience Letters, vol. 548, pp. 4-9, 2013.

[33] M. Wang, A. Zhou, and L. Xu, "The role of TLR4-mediated $\mathrm{PTEN} / \mathrm{PI} 3 \mathrm{~K} / \mathrm{AKT} / \mathrm{NF}-\kappa \mathrm{B}$ signaling pathway in neuroinflammation in hippocampal neurons," Neuroscience, vol. 269, pp. 93-101, 2014.

[34] S. Kawashima, T. Yamashita, Y. Miwa et al., "HMG-CoA reductase inhibitor has protective effects against stroke events in stroke-prone spontaneously hypertensive rats," Stroke, vol. 34, no. 1, pp. 157-163, 2003.

[35] X.-L. Chen, S. E. Varner, A. S. Rao et al., "Laminar flow induction of antioxidant response element-mediated genes in endothelial cells," Journal of Biological Chemistry, vol. 278, no. 2, pp. 703-711, 2003.

[36] S. Yun, A. Dardik, M. Haga et al., "Transcription factor Sp1 phosphorylation induced by shear stress inhibits membrane type 1-matrix metalloproteinase expression in endothelium," Journal of Biological Chemistry, vol. 277, no. 38, pp. 3480834814, 2002.

[37] A. Dardik, L. Chen, J. Frattini et al., "Differential effects of orbital and laminar shear stress on endothelial cells," Journal of Vascular Surgery, vol. 41, no. 5, pp. 869-880, 2005.

[38] T. Nagel, N. Resnick, and C. F. Dewey, "Vascular endothelial cells respond to spatial gradients in fluid shear stress by enhanced activation of transcription factors," Arteriosclerosis, Thrombosis, and Vascular Biology, vol. 19, no. 8, pp. 18251834, 1999.

[39] P. Nigro, J.-I. Abe, and B. C. Berk, "Flow shear stress and atherosclerosis: a matter of site specificity," Antioxidants \& Redox Signaling, vol. 15, no. 5, pp. 1405-1414, 2011.

[40] V. Sudhahar, S. A. Kumar, and P. Varalakshmi, "Role of lupeol and lupeol linoleate on lipemic-oxidative stress in experimental hypercholesterolemia," Life Sciences, vol. 78, no. 12, pp. 1329-1335, 2006. 
[41] Z. Zhang, C. Xu, J. Hao et al., "Beneficial consequences of Lupeol on middle cerebral artery-induced cerebral ischemia in the rat involves Nrf2 and P38 MAPK modulation," Metabolic Brain Disease, vol. 35, no. 5, pp. 841-848, 2020.

[42] H. Badshah, T. Ali, S.-U. Rehman et al., "Protective effect of lupeol against lipopolysaccharide-induced neuroinflammation via the $\mathrm{p} 38 / \mathrm{c}$-Jun N-terminal kinase pathway in the adult mouse brain," Journal of Neuroimmune Pharmacology, vol. 11, no. 1, pp. 48-60, 2016.

[43] L. Yang, J. Liu, and Y. Li, "Bornyl acetate suppresses ox-LDLinduced attachment of THP-1 monocytes to endothelial cells," Biomedicine \& Pharmacotherapy, vol. 103, pp. 234-239, 2018.

[44] M. J. Haley, G. Mullard, K. A. Hollywood, G. J. Cooper, W. B. Dunn, and C. B. Lawrence, "Adipose tissue and metabolic and inflammatory responses to stroke are altered in obese mice," Disease Models \& Mechanisms, vol. 10, no. 10, pp. 1229-1243, 2017.

[45] K. H. Nam, S. A. Yi, J. Lee et al., "Eudesmin impairs adipogenic differentiation via inhibition of S6K1 signaling pathway," Biochemical and Biophysical Research Communications, vol. 505, no. 4, pp. 1148-1153, 2018.

[46] H. K. Jo, G. W. Kim, K. J. Jeong, D. Y. Kim, and S. H. Chung, "Eugenol ameliorates hepatic steatosis and fibrosis by downregulating SREBP1 gene expression via AMPK-mTORp70S6K signaling pathway," Biological and Pharmaceutical Bulletin, vol. 37, no. 8, pp. 1341-1351, 2014.

[47] J. H. Choi, S. W. Kim, R. Yu, and J. W. Yun, "Monoterpene phenolic compound thymol promotes browning of 3T3-L1 adipocytes," European Journal of Nutrition, vol. 56, no. 7, pp. 2329-2341, 2017.

[48] Y. Li, L. Xu, K. Zeng et al., "Propane-2-sulfonic acid octadec9-enyl-amide, a novel PPAR $\alpha / \gamma$ dual agonist, protects against ischemia-induced brain damage in mice by inhibiting inflammatory responses," Brain, Behavior, and Immunity, vol. 66, pp. 289-301, 2017.

[49] B. De Filippis, A. Giancristofaro, A. Ammazzalorso et al., "Discovery of gemfibrozil analogues that activate PPAR $\alpha$ and enhance the expression of gene CPT1A involved in fatty acids catabolism," European Journal of Medicinal Chemistry, vol. 46, no. 10, pp. 5218-5224, 2011.

[50] H.-J. Jun, J. H. Lee, J. Kim et al., "Linalool is a PPAR $\alpha$ ligand that reduces plasma TG levels and rewires the hepatic transcriptome and plasma metabolome," Journal of Lipid Research, vol. 55, no. 6, pp. 1098-1110, 2014.

[51] A. M. Barrera-Sandoval, E. Osorio, and G. P. CardonaGómez, "Microglial-targeting induced by intranasal linalool during neurological protection postischemia," European Journal of Pharmacology, vol. 857, p. 172420, 2019.

[52] Y. P. He, Y. Z. Chen, R. M. Fang et al., "Effect of acorus tatarinowii and borneol on platelet activation in hyperlipidemia rats," Chinese Journal of Traditional Medical Science and Technology, vol. 6, pp. 337-338, 2003.

[53] J. Poplawski, B. Lozowicka, A. T. Dubis et al., "Synthesis and hypolipidemic and antiplatelet activities of alpha-asarone isomers in humans (in vitro), mice (in vivo), and rats (in vivo)," Journal of Medicinal Chemistry, vol. 43, no. 20, pp. 3671-3676, 2000.

[54] S. Chilmonczyk, "Activation of platelet function through G protein-coupled receptors," Circulation Research, vol. 99, no. 12, pp. 1293-1304, 2006.

[55] A. Yan, T. Zhang, and X. Yang, "Thromboxane A2 receptor antagonist SQ29548 reduces ischemic stroke-induced microglia/macrophages activation and enrichment, and ameliorates brain injury," Science Reports, vol. 6, p. 35885, 2016.

[56] D. Yu, K. E. Huang, and Q. D. Wu, "Study on anticoagulation mechanism of $\beta$-asarone based on network pharmacology," Traditional Chinese Drug Research \& Clinical Pharmacology, vol. 31, no. 3, pp. 324-329, 2020.

[57] I. D. Grice, K. L. Rogers, and L. R. Griffiths, "Isolation of bioactive compounds that relate to the anti-platelet activity of cymbopogon ambiguus," Evidence Based Complementary and Alternative Medicine, vol. 2011, Article ID 467134, 2011.

[58] C. Y. Min, H. Q. Liu, and F. Zhan, "Effect of cortex moutan on PGI2, TXA2, ET and NO in diabetic rats," Journal of Chinese Medicinal Materials, vol. 30, no. 6, pp. 687-690, 2007.

[59] B.-G. Wang, X. Hong, L. Li, J. Zhou, and X.-J. Hao, "Chemical constituents of two Chinese magnoliaceae plants, tsoongiodendron odorum and manglietiastrum sinicum, and their inhibition of platelet aggregation," Planta Medica, vol. 66, no. 6, pp. 511-515, 2000.

[60] T. Sairaren, A. Ristimäki, M.-L. Karjalainen-Lindsberg, A. Paetau, M. Kaste, and P. J. Lindsberg, "Cyclooxygenase-2 is induced globally in infarcted human brain," Annals of Neurology, vol. 43, no. 6, pp. 738-747, 1998.

[61] S. Nogawa, F. Zhang, M. E. Ross, and C. Iadecola, "Cyclooxygenase-2 gene expression in neurons contributes to ischemic brain damage," The Journal of Neuroscience, vol. 17, no. 8, pp. 2746-2755, 1997.

[62] W. Li, S. Wu, R. W. Hickey, M. E. Rose, J. Chen, and S. H. Graham, "Neuronal cyclooxygenase-2 activity and prostaglandins PGE2, PGD2, and PGF2 $\alpha$ exacerbate hypoxic neuronal injury in neuron-enriched primary culture," $\mathrm{Neu}$ rochemical Research, vol. 33, no. 3, pp. 490-499, 2008.

[63] T. Yagami, K. Ueda, K. Asakura et al., "Human group IIA secretory phospholipase A2 induces neuronal cell death via apoptosis," Molecular Pharmacology, vol. 61, no. 1, pp. 114-126, 2002.

[64] S. A. Saeed, R. U. Simjee, G. Shamim, and A. H. Gilani, "Eugenol: a dual inhibitor of platelet-activating factor and arachidonic acid metabolism," Phytomedicine, vol. 2, no. 1, pp. 23-28, 1995.

[65] H.-S. Chae, O.-H. Kang, Y.-S. Lee et al., "Inhibition of LPSinduced iNOS, COX-2 and inflammatory mediator expression by paeonol through the MAPKs inactivation in RAW 264.7 cells," The American Journal of Chinese Medicine, vol. 37, no. 1, pp. 181-194, 2009.

[66] S. Y. Park, S. J. Park, N. J. Park, W. H. Joo, S.-J. Lee, and Y.-W. Choi, " $\alpha$-Iso-cubebene exerts neuroprotective effects in amyloid beta stimulated microglia activation," Neuroscience Letters, vol. 555, pp. 143-148, 2013.

[67] J. Thevenet, A. Angelillo-Scherrer, M. Price, and L. Hirt, "Coagulation factor Xa activates thrombin in ischemic neural tissue," Journal of Neurochemistry, vol. 111, no. 3, pp. 828-836, 2009.

[68] E. S. Stein, Z. Itsekson-Hayosh, and A. Aronovich, "Thrombin induces ischemic LTP (iLTP): implications for synaptic plasticity in the acute phase of ischemic stroke," Science Reports, vol. 5, p. 7912, 2015.

[69] S. Dolati, M. Ahmadi, M. Khalili et al., "Peripheral Th17/Treg imbalance in elderly patients with ischemic stroke," Neurological Sciences, vol. 39, no. 4, pp. 647-654, 2018.

[70] H. Namdari, M. Izad, F. Rezaei, and Z. Amirghofran, "Thymol as a reciprocal regulator of $\mathrm{T}$ cell differentiation: promotion of regulatory $\mathrm{T}$ cells and suppression of Th1/Th17 cells," International Immunopharmacology, vol. 67, pp. 417-426, 2019. 
[71] L. Xue, H. Chen, K. Lu, J. Huang, H. Duan, and Y. Zhao, "Clinical significance of changes in serum neuroglobin and HIF- $1 \alpha$ concentrations during the early-phase of acute ischemic stroke," Journal of the Neurological Sciences, vol. 375, pp. 52-57, 2017.

[72] L. Huang, E. M. Kan, and J. Lu, "Toll-like receptor 4 mediates microglial activation and production of inflammatory mediators in neonatal rat brain following hypoxia: role of TLR4 in hypoxic microglia," Journal of Neuroinflammation, vol. 10, p. 23, 2013.

[73] W. Y. Liao, T. H. Tsai, T. Y. Ho et al., "Neuroprotective effect of paeonol mediates anti-inflammation via suppressing tolllike receptor 2 and toll-like receptor 4 signaling pathways in cerebral ischemia-reperfusion injured rats," Evidence Based Complementary and Alternative Medicine, vol. 2016, Article ID 3704647, 2016.

[74] B. L. Wang, L. Xuan, S. J. Dai et al., "Protective effect of $\beta$-asarone on $\mathrm{AD}$ rat model induced by intracerebroventricular injection of $\mathrm{A} \beta_{1-42}$ combined 2-VO and its mechanism," China Journal of Chinese Material and Media, vol. 42, no. 24, pp. 4847-4854, 2017.

[75] H. Moon, M. J. Kim, H. J. Son et al., "Five hTRPA1 agonists found in indigenous Korean mint, agastache rugosa," PLoS One, vol. 10, no. 5, Article ID e0127060, 2015.

[76] M. C. Kweon, A. Morabito, M. Giustizieri et al., “TRPV1 channels are critical brain inflammation detectors and neuropathic pain biomarkers in mice," Nature Communications, vol. 8, p. 15292, 2017.

[77] Z.-H. Xu, C. Wang, T. Fujita, C.-Y. Jiang, and E. Kumamoto, "Action of thymol on spontaneous excitatory transmission in adult rat spinal substantia gelatinosa neurons," Neuroscience Letters, vol. 606, pp. 94-99, 2015.

[78] C. K. Chen, P. Y. Hsu, and T. M. Wang, "TRPV4 activation contributes functional recovery from ischemic stroke via angiogenesis and neurogenesis," Molecular Neurobiology, vol. 55, no. 5, pp. 4127-4135, 2018.

[79] P. Jie, Z. Hong, Y. Tian et al., "Activation of transient receptor potential vanilloid 4 induces apoptosis in hippocampus through downregulating PI3K/Akt and upregulating p38 MAPK signaling pathways," Cell Death \& Disease, vol. 6, no. 6, p. e1775, 2015. 